\title{
OS CINCO PRIMEIROS ANOS DE PROGRAMA GESTÃO PÚBLICA E CIDADANIA
}

Patrícia Laczynski e Marta Ferreira Santos Farah

(Membros da Equipe de Coordenação do Programa GESTÃO PÚBLICA E CIDADANIA)

O Programa GESTÃO PÚBLICA E CIDADANIA tem o objetivo de focalizar o que está indo bem na administração pública, descobrir as inovações e os inovadores, e aumentar o estoque de conhecimentos sobre experiências alternativas em gestão pública. Com cinco anos de experiência, foi possivel formar um banco de dados com 3.391 programas, projetos e atividades de instituições públicas governamentais dos níveis estadual e municipal e das organizações próprias dos povos indígenas. Essas experiências foram inscritas nos Ciclos de Premiação anuais que ocorreram de 1996 a 2000. Neste último ano, o Programa GESTÃo PÚBLICA E CIDADANIA atinge o maior número de programas, projetos e atividades inscritos desde a sua criação: 946 .

TABELA 1 - DISTRIBUIÇÃO DOS PROGRAMAS, PROJETOS E ATIVIDADES INSCRITOS, POR NIVEL DE GOVERNO - CICLOS DE PREMIACCÃO 1996, 1997, 1998, 1999 E 2000

\begin{tabular}{|c|c|c|c|c|c|c|c|c|c|c|c|c|}
\hline \multirow{2}{*}{ nivel de governo } & \multicolumn{2}{|c|}{ Total } & \multicolumn{2}{|c|}{1996} & \multicolumn{2}{|c|}{1997} & \multicolumn{2}{|c|}{1998} & \multicolumn{2}{|c|}{1999} & \multicolumn{2}{|c|}{2000} \\
\hline & n.a. & $(\%)$ & n.a. & $(\%)$ & n.a. & $(\%)$ & n.a. & $(\%)$ & n.a. & $(\%)$ & n.a. & $(\%)$ \\
\hline total & 3.391 & 100,0 & 629 & 100,0 & 297 & 100,0 & 631 & 100,0 & 888 & 100,0 & 946 & 100,0 \\
\hline estadual (1) & 760 & 22,4 & 134 & 21,3 & 122 & 41,1 & 224 & 35,5 & 157 & 17,7 & 123 & 13,0 \\
\hline municipal ${ }^{(2)}$ & 2.597 & 76,6 & 488 & 77,6 & 174 & 58,6 & 400 & 63,4 & 721 & 81,2 & 814 & 86,0 \\
\hline indigena & 34 & 1,0 & 7 & 1,1 & 1 & 0.3 & 7 & 1,1 & 10 & 1,1 & 9 & 1,0 \\
\hline $\begin{array}{l}\text { (1) } \\
\text { (2) }\end{array}$ & & & & & & & & & & & & \\
\hline
\end{tabular}

Desse total, a maior parte dos programas, projetos e atividades são provenientes de governos municipais - 86,0\%, em 2000 e 76,6\%, nos cinco anos (ver tabela 1). Ao longo dos cinco anos de Programa, foram 556 municipios que inscreveram alguma iniciativa, o que representa cerca de $10 \%$ do total de municípios brasileiros. De 1999 para 2000, houve um acréscimo de 12,9\% no número de inscrições de governos municipais (ver tabela 2). Isso explica-se, em parte, porque, sendo 2000 ○ último ano de gestão, houve mais tempo para os programas, projetos e atividades serem consolidados. Por outro lado, em período pré-eleitoral tende a haver maior interesse em divulgar as realizações da gestão que se encerra.

Em contrapartida, as inscrições estaduais diminuíram 21,7\% (ver tabela 2), passando de 157 inscrições, em 1999, para 123, em 2000.

Em 2000, as iniciativas das organizações próprias dos povos indígenas representam 1,0\% do total, mantendo a média dos outros anos (ver tabela 1). Desde 1997, quando as inscrições diminuíram 
de sete para uma, a coordenação do Programa tem buscado divulgar entre os povos indígenas o Ciclo de Premiação. Para tanto elaborou, em parceria com o Núcleo de Cultura Indígena, um folder com linguagem específica e um calendário "aberto" (quando as suas inscrições não cumprem o prazo determinado pelo Programa GESTÃO PÚBLICA E CIDADANIA, elas são validadas automaticamente para o Ciclo de Premiação do ano seguinte), viabilizando a maior participação destas iniciativas.

TABELA 2 - VARIAÇÃO PERCENTUAL DO NÚMERO DE INSCRIÇÕES POR NÍVEL DE GOVERNO CICLOS DE PREMIACÃO 1996, 1997, 1998 E 1999

\begin{tabular}{l|r|r|r|r}
\hline nível de governo & $\begin{array}{c}\text { De 1996 para } \\
1997\end{array}$ & $\begin{array}{c}\text { de 1997 para } \\
1998\end{array}$ & $\begin{array}{c}\text { de 1998 para } \\
1999\end{array}$ & $\begin{array}{c}\text { de } 1999 \text { para } \\
2000\end{array}$ \\
\hline total & $-52,8$ & $+112,5$ & $+40,7$ & $+6,5$ \\
$\quad$ estadual & $-9,0$ & $+83,6$ & $-29,9$ & $-21,7$ \\
$\quad$ municipal (1) & $-64,3$ & $+129,9$ & $+80,3$ & $+12,9$ \\
\hline \multicolumn{7}{c}{ Inclui iniciativas intermunicipais. } \\
\hline
\end{tabular}

No Ciclo de Premiação 2000, 20 governos estaduais inscreveram programas, projetos e atividades. Em 1999, foram 19 governos estaduais e, em 1998, 21. Em 2000, seis governos estaduais - DF, MS, PB, PE, RR e TO - que não haviam inscrito nenhuma iniciativa estadual no ano anterior, participaram do Ciclo de Premiação.

Em relação à participação de governos locais, 254 municípios inscreveram iniciativas (municipais ou intermunicipais) em 2000. Nos anos anteriores, o número de municípios, cujos governos municipais inscreveram iniciativas, tinha sido de 235 (1999) e 140 (1998), o que confirma a presença crescente da participação de municípios no Programa.

Observa-se que o Programa GESTÃo PÚBLICA E CIDADANIA, foi ampliando a sua penetração junto a estados e municípios, de forma que a cada ano novas localidades têm passado a participar do Ciclo anual, inscrevendo alguns programas, projetos ou atividades. Assim, no que se refere ao nível estadual de governo, há apenas um estado que nunca participou do Programa: o Estado de Alagoas.

Da mesma forma, em relação aos municípios, observa-se que a cada ano um grupo de municípios que nunca havia participado do Programa GESTÃO PÚBLICA E CIDADANIA, passa a inscrever experiências em 2000. Assim, neste ano, 114 governos municipais que nunca haviam enviado inscrições, passaram a participar (nos anos anteriores, o número de novos municípios participantes foram: 1997 - 52; 1998 - 98; 1999 - 130).

A maior parte das inscrições é da região Sudeste $(46,0 \%)$, como vinha ocorrendo nos últimos anos (ver tabela 3). Mas o que mais destaca neste ano é o crescimento da participação da região Sul (de 29,6\% em 1999 para 35,2\% em 2000). Este crescimento, no entanto, deve-se sobretudo à 
presença da inscrição de um número elevado de programas, projetos e atividades por um mesmo municipio.

Os municípios cujos governos mais inscreveram programas, projetos e atividades foram Campo Mourão - PR (48), Belo Horizonte - MG (35), Paranavaí - PR (34), Quitandinha - PR (27), São José dos Campos - SP (21), Porto Alegre - RS (20), Camarigibe - PE (17), Presidente Prudente - SP (15), Campinas - SP (13), Joinville - SC (13), Santo André - SP (13), Curitiba - PR (12), Blumenau - SC (11), João Monlevade - MG (11), Betim - MG (10), Jaraguá do Sul - SC (10), Ribeirão Pires - SP (10) e Uberlândia - MG (10). Com exceção de Camaragibe, todos estes municípios são das regiões Sudeste e Sul.

Constata-se que, dentre os municípios com maior número de inscrições (mais de 10 inscrições), há oito da região Sul, que totalizam sozinhos 175 iniciativas inscritas. Fato similar ocorre na região Sudeste. Assim, das localidades com mais de 10 inscrições, nove são desta região, totalizando 138 iniciativas. A presença da região Nordeste sofre menos a influência desta inscrição de "pacotes" de programas. Apenas um município desta região inscreveu mais de 10 programas, projetos ou atividades.

TABELA 3 - DISTRIBUIÇÃO DOS PROGRAMAS, PROJETOS E ATIVIDADES INSCRITOS, POR REGIÃO GEOGRÁFICA - CICLOS DE PREMIAC̄̃̃O 1996, 1997, 1998, 1999 E 2000

\begin{tabular}{|c|c|c|c|c|c|c|c|c|c|c|c|c|}
\hline \multirow[t]{2}{*}{ Região } & \multicolumn{2}{|c|}{ total } & \multicolumn{2}{|c|}{1996} & \multicolumn{2}{|c|}{1997} & \multicolumn{2}{|c|}{1998} & \multicolumn{2}{|c|}{1999} & \multicolumn{2}{|c|}{2000} \\
\hline & n.a. & $(\%)$ & n.a. & $(\%)$ & n.a. & $(\%)$ & n.a. & $(\%)$ & n.a. & $(\%)$ & n.a. & $(\%)$ \\
\hline otal & 3.391 & 100,0 & 629 & 100,0 & 297 & 100,0 & 631 & 100,0 & 888 & 100,0 & 946 & 100,0 \\
\hline Norte & 110 & 3,2 & 17 & 2,7 & 8 & 2,7 & 26 & 4,1 & 38 & 4,3 & 21 & 2,2 \\
\hline Nordeste & 428 & 12,6 & 70 & 11,1 & 61 & 20,5 & 60 & 9,5 & 116 & 13,1 & 121 & 12,8 \\
\hline Sudeste & 1.716 & 50,6 & 384 & 61,1 & 120 & 40,4 & 324 & 51,4 & 453 & 51,0 & 435 & 46,0 \\
\hline Sul & 960 & 28,3 & 134 & 21,3 & 62 & 20,9 & 168 & 26,6 & 263 & 29,6 & 333 & 35,2 \\
\hline Centro-Oeste & 177 & 5,2 & 24 & 3,8 & 46 & 15,5 & 53 & 8,4 & 18 & 2,0 & 36 & 3,8 \\
\hline
\end{tabular}

Assim, é interessante rever a participação regional, para o caso dos programas municipais, considerando a participação de municípios e não o número de inscrições. Nesse caso, no Ciclo de Premiação 2000, a participação do Nordeste aumenta de 12,8\% (porcentagem de programas, projetos e atividades por região) para $15 \%$ (porcentagem da participação de governos municipais por região). Mas a participação das regiões Sudeste e Sul continua sendo significativamente mais expressiva (ver tabela 4). 
TABELA 4 - DISTRIBUIÇÃO DOS MUNICIPIOS PARTICIPANTES (1),

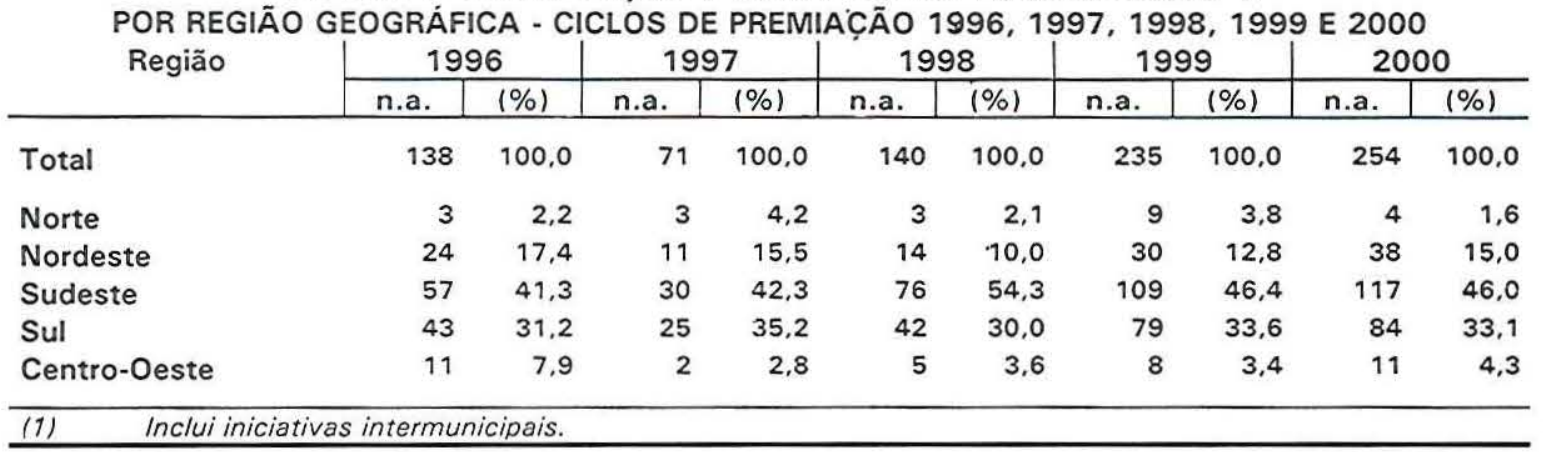

Como já mencionado antes, em todos estes cinco anos, foram os governos municipais que mais inscreveram iniciativas no Programa GESTÃO PÚBLICA E CIDADANIA. Se cruzarmos as participações estaduais, municipais e indígenas com as regiões geográficas brasileiras, percebemos que a diferença entre a participação Sul e Nordeste, no que diz respeito às iniciativas estaduais, não é grande (ver tabela 5). A região Centro-Oeste apresenta também uma maior participação quando se trata de programas, projetos e atividades estaduais. Isso se deve a um grande número de inscrições de experiências do Distrito Federal em anos anteriores.

As inscrições indígenas se concentram na região Norte, área brasileira onde existem várias comunidades de povos indígenas. Interessante notar a pouca participação das comunidades indígenas da região Centro-Oeste, outra área geográfica onde habita grande número de povos indígenas.

TABELA 5 - PROGRAMAS, PROJETOS E ATIVIDADES INSCRITOS, POR NIVEL DE GOVERNO, SEGUNDO A REGIÃO GEOGRÁFICA - TODOS OS CICLO DE PREMIACCÃO (1996 A 2000)

\begin{tabular}{|c|c|c|c|c|c|c|}
\hline \multirow[b]{2}{*}{ ÁREAS DE GOVERNO } & \multicolumn{2}{|c|}{ estadual $^{(1)}$} & \multicolumn{2}{|c|}{ municipal I $^{(2)}$} & \multicolumn{2}{|c|}{ indigena } \\
\hline & n.a. & $(\%)$ & n.a. & $(\%)$ & n.a. & $(\%)$ \\
\hline total & 763 & 100,0 & 2.595 & 100,0 & 33 & 100,0 \\
\hline Norte & 48 & 6,3 & 45 & 1,7 & 21 & 63,6 \\
\hline Nordeste & 117 & 15,3 & 299 & 11,5 & -- & 0.0 \\
\hline Sudeste & 363 & 47,6 & 1.346 & 51,9 & 9 & 27,3 \\
\hline Sul & 136 & 17,8 & 821 & 31,6 & -- & 0,0 \\
\hline Centro-Oeste & 99 & 13,0 & 84 & 3,2 & 3 & 9,1 \\
\hline $\begin{array}{l}\text { Inclui iniciativas } \\
\text { Inclui iniciativas }\end{array}$ & do Distrit & ederal. & & & & \\
\hline
\end{tabular}

Ainda a respeito do número de iniciativas inscritas por municípios, é interessante notar como tem crescido o número de municípios que inscreveram apenas uma ou duas experiências nos Ciclos de Premiação. De 1999 para 2000, conforme a tabela 6, este número subiu de 166 para 170 
TABELA 6 - MUNICÍPIOS QUE ENVIARAM APENAS UMA OU DUAS INSCRIÇÕES, POR NIVEL DE GOVERNO - CICLOS DE PREMIACCÃO 1999 E 2000

\begin{tabular}{l|c|c|c|c}
\hline número de inscrições & \multicolumn{2}{|c|}{1999} & \multicolumn{2}{c}{2000} \\
\cline { 2 - 5 } & mun. & $\%$ & mun. & $\%$ \\
\hline uma inscrição & 124 & 52,8 & 146 & 57,3 \\
duas inscrições & 42 & 17,9 & 24 & 9,4 \\
$\quad$ mais de duas & 69 & 29,4 & 85 & 33,3 \\
inscrições & & & & 100 \\
total & 235 & 100,0 & 254 & 100 \\
\hline
\end{tabular}

No total, em 2000, foram 254 municípios que inscreveram programas, projetos ou atividades, 8,1\% a mais que em 1999 (235 municípios), conforme a tabela 4. É interessante notar que o grupo de municipios de população entre 50.000 e 500.000 habitantes que tem uma participação no Programa é maior do que em relação ao número total de municípios do pais (43,6\% de inscrições em 2000 versus $9,5 \%$ de todos os municípios brasileiros). Contudo há um número considerável de inscrições provenientes de municípios com menos de 20.000 habitantes, normalmente considerados com poucos recursos (ver tabela 7).

TABELA 7 - DISTRIBUIÇÃO DOS MUNICIPIOS COM INSCRIC̣ÕES NO PROGRAMA - CICLOS DE PREMIACÃO 1999 E 2000

\begin{tabular}{|c|c|c|c|c|c|}
\hline \multirow{2}{*}{$\begin{array}{l}\text { número habitantes } \\
\text { (em milhares) }\end{array}$} & \multicolumn{2}{|c|}{$1999^{(1)}$} & \multicolumn{2}{|c|}{$2000^{(1)}$} & \multirow{2}{*}{$\begin{array}{c}\text { IBGE }^{(2)} \\
(\%) \\
\end{array}$} \\
\hline & n.a. & $(\%)$ & n.a. & $(\%)$ & \\
\hline total & 235 & 100 & 254 & 100,0 & 100,0 \\
\hline até 20 & 71 & 30,2 & 72 & 28,4 & 71,0 \\
\hline+ de 20 até 50 & 48 & 20,5 & 43 & 16,9 & 19,0 \\
\hline+ de 50 até 100 & 36 & 15,3 & 50 & 19,7 & 5,8 \\
\hline+ de 100 até 200 & 29 & 12,3 & 28 & 11,0 & 2,3 \\
\hline+ de 200 até 500 & 29 & 12,3 & 33 & 13,0 & 1,4 \\
\hline+ de 500 até 1.000 & 9 & 3,8 & 9 & 3,5 & 0,3 \\
\hline+ de 1.000 até 5.000 & 6 & 2,6 & 7 & 2,8 & 0,2 \\
\hline+ de 5.000 & 2 & 0,9 & 2 & 0,8 & 0,02 \\
\hline sem informação & 5 & 2,1 & 10 & 3,9 & $-\cdot$ \\
\hline $\begin{array}{l}\text { Inclui iniciativa } \\
\text { População dos }\end{array}$ & gun & OE - & & & \\
\hline
\end{tabular}

A maior parte dos programas, projetos e atividades inscritos teve seu início na própria gestão dos governos responsáveis pelas inscrições (ver tabela 8). Ao longo dos últimos quatro Ciclos de Premiação (não foi possivel fazer este levantamento para o ano de 1996), 57,8\% das inscrições foram implementadas durante a gestão do governo responsável pela inscrição, $30,2 \%$ durante a gestão antecessora e $11,9 \%$ antes da gestão antecessora. É preciso cuidado ao fazer inferências sobre o padrão de continuidade de políticas públicas a partir destes resultados. Podemos, no entanto, levantar a hipótese de que os governos se sentem mais estimulados a inscreverem 
programas implementados por sua própria iniciativa, particularmente em ano pré-eleitoral, quando muitos programas estão consolidados.

Mas é importante ressaltar que estudo realizado em 1997, após mudança de governos municipais, por Spink, Clemente e Keppke (1999) junto a 274 programas, projetos e atividades inscritos no Ciclo de Premiação 1996, apurou que $88,0 \%$ deles haviam continuado no governo seguinte, mesmo com a mudança de partido político no poder em metade dos casos.

TABELA 8 - PROGRAMAS, PROJETOS E ATIVIDADES INSCRITOS POR NIVEL DE GOVERNO SEGUNDO O PERIODO DE IMPLEMENTACCÃO - CICLOS DE PREMIAÇÃO 1997, 1998, 1999 E 2000 (*)

\begin{tabular}{|c|c|c|c|c|c|c|c|c|c|c|c|c|c|c|c|}
\hline \multirow{3}{*}{$\begin{array}{l}\text { GESTÃO GOVERNAMENTAL } \\
\text { RESPONSÁVEL PELA } \\
\text { INSCRICĀO }\end{array}$} & \multicolumn{15}{|c|}{ ANO DO CICLO DE PREMIAC̣ÃO E NIVEL DE GOVERNO } \\
\hline & \multicolumn{3}{|c|}{ TOTAL } & \multicolumn{3}{|c|}{1997} & \multicolumn{3}{|c|}{1998} & \multicolumn{3}{|c|}{1999} & \multicolumn{3}{|c|}{2000} \\
\hline & Total & est. & mun. & total & est. & nun. & total & est. & mun. & otal & est. & mun. & otal & \begin{tabular}{l|l} 
est. & $r$
\end{tabular} & mun. \\
\hline TOTAL & 2735 & 631 & 2108 & 296 & 125 & 171 & 624 & 224 & 400 & 878 & 157 & 721 & 937 & 123 & 814 \\
\hline gestăo & 1582 & 266 & 1320 & 85 & 85 & $\ldots(2)$ & 343 & 160 & 183 & 518 & -- (2) & 518 & 636 & 19 & 617 \\
\hline gest & 827 & 254 & 573 & 159 & 30 & 129 & 199 & 37 & 162 & 248 & 104 & 144 & 221 & 83 & 138 \\
\hline an & 326 & 111 & 215 & 52 & 10 & 42 & 82 & 27 & 55 & 112 & 53 & 59 & 80 & 21 & 59 \\
\hline DISTRIBUICCÃO PERC & 100 & 100 & 100 & 100 & 100 & 100 & 100 & 100 & 100 & 100 & 100 & 100 & 100 & 100 & 10 \\
\hline gestão at & 57,8 & 42,2 & 62,6 & 28,7 & 68,0 & $\ldots(2)$ & 55,0 & 71,4 & 45,8 & 59,0 & -. (2) & 71,8 & 67,9 & 15,4 & 10,8 \\
\hline gestăo antecessora & 30,2 & 40,3 & 27,2 & 53.7 & 24,0 & 75,4 & 31,9 & 16,5 & 40,5 & 28,2 & 66,2 & 20,0 & 23,6 & 67,5 & 17,0 \\
\hline ante & 11,9 & 17,6 & 10,2 & 6 & 8,0 & 24,6 & 1 & 12,1 & 13,7 & 2,8 & 33,8 & 8,2 & 8,5 & 17,1 & 7. \\
\hline & & & & & & & & & & & & & & & \\
\hline 1) & uais & & itnis & & & & d & & 1 & & & & & 99): & \\
\hline Náo se aplica porqu & Prog & & kige 7 & & ti & fun & 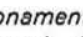 & & & & & & & & \\
\hline Para os governos & taduais, & aus & iénio & 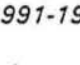 & 004 & & $d$ & 199 & 1 & 19 & lpara & 900 & $d e$ & & 8 \\
\hline Para os governos & duais, & até & 90 & 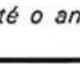 & de & 98 & até & 1994 & ara & no & 199 & l: pars & ra os & & 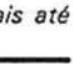 \\
\hline
\end{tabular}

Solicita-se a cada órgão público responsável pelos programas, projetos e atividades que classifique sua iniciativa segundo áreas e subáreas de atuação governamental (ver quadro II, p. 19). As áreas são Administração e Governo, Infra-estrutura e Meio Ambiente, Serviços Públicos, Cidadania e Direitos Humanos, Desenvolvimento Econômico e Social, Legislativo e Judiciário.

Nos cinco anos de Ciclos de Premiação, a área Serviços Públicos foi a campeã em inscrições, seguida das áreas Cidadania e Direitos Humanos e Administração e Governo (ver tabela 9). Já em 2000, a terceira área com número de inscrições foi Desenvolvimento Econômico e Social. 
TABELA 9 - DISTRIBUIC̣ÃO DOS PROGRAMAS, PROJETOS E ATIVIDADES SEGUNDO AS GRANDES ÁREAS DE ACCÃO GOVERNAMENTAL - TODOS OS CICLOS DE PREMIACCÃO (1996 A 2000)

\begin{tabular}{|c|c|c|c|c|c|c|c|c|c|c|c|c|c|}
\hline \multirow{2}{*}{\multicolumn{2}{|c|}{ áreas de governo }} & \multicolumn{2}{|c|}{ total } & \multicolumn{2}{|c|}{1996} & \multicolumn{2}{|c|}{1997} & \multicolumn{2}{|c|}{1998} & \multicolumn{2}{|c|}{1999} & \multicolumn{2}{|c|}{2000} \\
\hline & & n.a. & $(\%)$ & n.a. & $(\%)$ & n.a. & $(\%)$ & n.a. & $(\%)$ & n.a. & $(\%)$ & n.a. & $(\%)$ \\
\hline tot & & 3.391 & 100,0 & 629 & 100,0 & 297 & 100,0 & 631 & 100,0 & 888 & 100,0 & 946 & 100,0 \\
\hline ser & públicos & 1.530 & 45,1 & 280 & 44,5 & 144 & 48,5 & 258 & 40,9 & 401 & 45,1 & 447 & 47,3 \\
\hline cid & & 806 & 23,8 & 103 & 16,4 & 51 & 17,2 & 171 & 27,1 & 228 & 25,7 & 253 & 26,7 \\
\hline$a d r$ & ração $o^{|4|}$ & 373 & 11,0 & 108 & 17,2 & 28 & 9,4 & 78 & 12,4 & 90 & 10,1 & 69 & 7,3 \\
\hline des & lvimento ${ }^{(2)}$ & 347 & 10,2 & 64 & 10,2 & 42 & 14,1 & 68 & 10.8 & 85 & 9,6 & 88 & 9,3 \\
\hline inf & rutura ${ }^{\mid 3]}$ & 310 & 9,1 & 65 & 10,3 & 31 & 10,4 & 51 & 8,1 & 77 & 8,7 & 86 & 9,1 \\
\hline jud & & 17 & 0,5 & 7 & 1,1 & 1 & 0,3 & 3 & 0,5 & 4 & 0,5 & 2 & 0,2 \\
\hline leg & & 8 & 0,2 & 2 & 0,3 & -- & - & 2 & 0,3 & 3 & 0,3 & 1 & 0,1 \\
\hline $\begin{array}{l}(1) \\
(2) \\
(3) \\
(4) \\
\end{array}$ & $\begin{array}{l}\text { Cidadania e } \\
\text { Desenvolvir } \\
\text { Infra-estrutu } \\
\text { Administrac }\end{array}$ & $\begin{array}{l}\text { direitos h } \\
\text { ento ecor } \\
\text { o e meio } \\
\text { o e gove }\end{array}$ & $\begin{array}{l}\text { manos. } \\
6 \text { mico e } \\
\text { ambiente } \\
\text { no. }\end{array}$ & cial. & & & & & & & & & \\
\hline
\end{tabular}

Em 2000, as áreas Serviços Públicos e Cidadania e Direitos Humanos abrangem a maior parte das inscrições - 74,0\% (ver tabela 10). É interessante notar que a área Infra-estrutura e Meio Ambiente teve sua representação aumentada ao longo do processo de seleção das iniciativas. Em relação ao total de programas, projetos e atividades inscritos, esta área participou com 9,1\%, enquanto em relação ao total de experiências pré-finalistas e finalistas, $20 \%$ das experiências são desta área.

TABELA 10 - DISTRIBUIÇÃO DAS INICIATIVAS INSCRITAS, PRÉ-FINALISTAS E FINALISTAS SEGUNDO AS GRANDES ÁREAS DE AÇÃO GOVERNAMENTAL - CICLO DE PREMIAC̄ÃO 2000

\begin{tabular}{|c|c|c|c|c|c|c|c|c|}
\hline \multirow[b]{2}{*}{ ÁREAS DE GOVERNO } & \multicolumn{2}{|c|}{ inscritos } & \multicolumn{2}{|c|}{ semifinalistas } & \multicolumn{2}{|c|}{ pré-finalistas } & \multicolumn{2}{|c|}{ finalistas } \\
\hline & n.a. & $(\%)$ & n.a. & $(\%)$ & n.a. & $(\%)$ & \begin{tabular}{l|l} 
n.a. & \\
\end{tabular} & $(\%)$ \\
\hline total & 946 & 100 & 100 & 100 & 30 & 100 & 20 & 100 \\
\hline serviços públicos & 447 & 47,3 & 29 & 29,0 & 9 & 30,0 & 8 & 40,0 \\
\hline cidadania e direitos humanos & 253 & 26,7 & 27 & 27,0 & 5 & 16,7 & 4 & 20,0 \\
\hline desenv. econômico e social (1) & 88 & 9,3 & 19 & 19,0 & 6 & 20,0 & 2 & 10,0 \\
\hline infra-estrutura e meio ambiente & 86 & 9,1 & 14 & 14,0 & 6 & 20,0 & 4 & 20,0 \\
\hline administração e governo & 69 & 7,3 & 10 & 10,0 & 4 & 13,3 & 2 & 10,0 \\
\hline judiciário & 2 & 0.2 & 1 & 1,0 & - & $\cdots$ & -- & - \\
\hline legislativo & 1 & 0,1 & -- & -- & -- & - & -- & -- \\
\hline
\end{tabular}

Se compararmos as inscrições dos programas, projetos e atividades inscritos nos cinco anos de Ciclos de Premiação, segundo as áreas de ação governamental, percebemos que a participação percentual das áreas de Serviços Públicos e Cidadania e Direitos Humanos diminui quando passa das inscrições municipais $(71,3 \%)$ para as estaduais $(61,0 \%)$, conforme tabela 11 . Já outras áreas, com mais tradição de responsabilidade estatal, esta participação aumenta. 
TABELA 11 - PROGRAMAS, PROJETOS E ATIVIDADES INSCRITOS, POR NIVEL DE GOVERNO, SEGUNDO AS GRANDES ÁREAS DE AÇÃO GOVERNAMENTAL - TODOS OS CICLOS DE PREMIACÃO (1996 a 2000)

\begin{tabular}{|c|c|c|c|c|c|c|}
\hline \multirow[b]{2}{*}{ ÁREAS DE GOVERNO } & \multicolumn{2}{|c|}{ estadual ${ }^{(1)}$} & \multicolumn{2}{|c|}{ municipal $^{(2)}$} & \multicolumn{2}{|c|}{ indígena } \\
\hline & n.a. & (\%) & n.a. & $(\%)$ & n.a. & $(\%)$ \\
\hline Total & 763 & 100,0 & 2.594 & 100,0 & 34 & 100,0 \\
\hline serviços públicos & 291 & 38,1 & 1234 & 47,6 & 6 & 17,6 \\
\hline cidadania e direitos humanos & 175 & 22,9 & 615 & 23,7 & 16 & 47,1 \\
\hline administração e governo & 95 & 12,5 & 277 & 10,7 & 1 & 2,9 \\
\hline desenvolvimento econômico e social & 102 & 13,4 & 238 & 9,2 & 6 & 17,6 \\
\hline infra-estrutura e meio ambiente & 82 & 10,7 & 223 & 8,6 & 5 & 14,7 \\
\hline Legislativo & 8 & 1,0 & 5 & 0,2 & 0 & 0,0 \\
\hline Judiciário & 10 & 1,3 & 2 & 0,1 & 0 & 0,0 \\
\hline $\begin{array}{l}\text { Inclui iniciativas interestadua } \\
\text { Inclui iniciativas intermunicip }\end{array}$ & rito of & & & & & \\
\hline
\end{tabular}

Dentro dessas áreas, as subáreas com mais inscrições são Educação lárea Serviços Públicos), Criança e Adolescente (área Cidadania e Direitos Humanos) e Saúde (área Serviços Públicos) - representando $48,2 \%$ do total de inscrições em 2000 e 42,5\% do total de inscrições dos cinco anos de Programa GESTÃO PÚBLICA E CIDADANIA (ver tabela 12). A presença expressiva dessas áreas é um indicativo da relevância destes temas na agenda atual dos governos locais, processo influenciado pela descentralização de recursos determinados pela Constituição de 1988 , associado a uma tendência de descentralização dos encargos nas áreas sociais.

TABELA 12 - DISTRIBUIÇÃO DOS PROGRAMAS, PROJETOS E ATIVIDADES INSCRITOS, POR ÁREAS DE ACÃO GOVERNAMENTAL - CICLOS DE PREMIACÃOO 1996, 1997, 1998, 1999 E 2000

\begin{tabular}{|c|c|c|c|c|c|c|c|c|c|c|c|c|}
\hline \multirow{2}{*}{$\begin{array}{l}\text { área de ação } \\
\text { governamental }\end{array}$} & \multicolumn{2}{|c|}{ total } & \multicolumn{2}{|c|}{1996} & \multicolumn{2}{|c|}{1997} & \multicolumn{2}{|c|}{1998} & \multicolumn{2}{|c|}{1999} & \multicolumn{2}{|c|}{2000} \\
\hline & n.a. & $(\%)$ & n.a. & $(\%)$ & n.a. & $(\%)$ & n.a. & $(\%)$ & n.a. & $(\%)$ & n.a. & $(\%)$ \\
\hline total & 3.391 & 100 & 629 & 100 & 297 & 100 & 631 & 100 & 888 & 100 & 946 & 100 \\
\hline educação & 537 & 15,8 & 98 & 15,6 & 60 & 20,2 & 74 & 11,7 & 122 & 13,7 & 183 & 19,3 \\
\hline criança e adolescente & 488 & 14,4 & 50 & 7,9 & 27 & 9,1 & 98 & 15,5 & 141 & 15,9 & 172 & 18,2 \\
\hline saúde & 416 & 12,3 & 81 & 12,9 & 45 & 15,2 & 75 & 11,9 & 114 & 12,8 & 101 & 10,7 \\
\hline assistência social & 180 & 5,3 & 18 & 2,9 & 6 & 2,0 & 26 & 4,1 & 70 & 7,9 & 60 & 6,3 \\
\hline $\begin{array}{l}\text { cultura, patrimônio histórico e } \\
\text { artistico(11) }\end{array}$ & 164 & 4,8 & 38 & 6,0 & 17 & 5,7 & 23 & 3,6 & 32 & 3,6 & 54 & 5.7 \\
\hline $\begin{array}{l}\text { formação de mão-de-obra, } \\
\text { geração de emprego e renda }\end{array}$ & 143 & 4,2 & 16 & 2,5 & 12 & 4,0 & 30 & 4,8 & 47 & 5.3 & 38 & 4,0 \\
\hline participação popular & 106 & 3,1 & 29 & 4,6 & 7 & 2,4 & 27 & 4,3 & 21 & 2,4 & 22 & 2,3 \\
\hline $\begin{array}{l}\text { desenvolvimento regional e } \\
\text { local em bases sustentáveis }\end{array}$ & 103 & 3,0 & 21 & 3,3 & 12 & 4,0 & 20 & 3,2 & 21 & 2,4 & 29 & 3,1 \\
\hline deficiente & 91 & 2,7 & 12 & 1,9 & 8 & 2,7 & 30 & 4,8 & 12 & 1,4 & 29 & 3,1 \\
\hline limpeza pública & 81 & 2,4 & 12 & 1,9 & 5 & 1,7 & 22 & 3,5 & 18 & 2,0 & 24 & 2,5 \\
\hline idoso & 79 & 2,3 & 10 & 1,6 & 6 & 2,0 & 16 & 2,5 & 32 & 3,6 & 15 & 1,6 \\
\hline lazer e esportes ${ }^{(1)}$ & 66 & 1,9 & 0 & 0,0 & 1 & 0,3 & 16 & 2,5 & 28 & 3,2 & 21 & 2,2 \\
\hline saneamento & 63 & 1,9 & 8 & 1,3 & 9 & 3,0 & 9 & 1,4 & 19 & 2,1 & 18 & 1,9 \\
\hline $\begin{array}{l}\text { segurança pública e } \\
\text { policiamento }\end{array}$ & 58 & 1,7 & 2 & 0,3 & 2 & 0.7 & 32 & 5,1 & 11 & 1,2 & 11 & 1,2 \\
\hline demais áreas & 816 & 24,1 & 234 & 37,2 & 80 & 26,9 & 133 & 21,1 & 200 & 22,5 & 169 & 17,9 \\
\hline
\end{tabular}


Se analisarmos o total de inscrições estaduais, municipais e indígenas, segundo as subáreas, percebemos pequenas diferenças entre as participações percentuais municipais e estaduais, salvo em casos como Deficiente e Segurança Pública e Policiamento (ver tabela 13). Em relação aos programas, projetos e atividades de organizações de povos indigenas, a área que mais concentra inscrições é Desenvolvimento Regional e Local em bases Sustentáveis (17,6\%). Uma outra área que apresenta muitas inscrições de iniciativas indígenas, mas poucas de iniciativas estaduais e municipais, é a subárea Minorias. Só em 1999, esta área abrangeu $90 \%$ das inscrições de povos indigenas.

TABELA 13 - PROGRAMAS, PROJETOS E ATIVIDADES INSCRITOS, POR NIVEL DE GOVERNO, SEGUNDO AS SUBÁREAS DE AC̣ÃO GOVERNAMENTAL - TODOS OS CICLOS DE PREMIAC̣ÃO

\begin{tabular}{|c|c|c|c|c|c|c|}
\hline \multirow[b]{2}{*}{ ÁREAS DE GOVERNO } & \multicolumn{2}{|c|}{ estadual ${ }^{(1)}$} & \multicolumn{2}{|c|}{ municipal $^{(2)}$} & \multicolumn{2}{|c|}{ indigena } \\
\hline & n.a. & $(\%)$ & n.a. & $(\%)$ & n.a. & $(\%)$ \\
\hline total & 763 & 100,0 & 2.594 & $i \cup 0,0$ & 34 & 100,0 \\
\hline educaçăo & 106 & 13,9 & 430 & 16,6 & 1 & 2,9 \\
\hline criança e adolescente & 74 & 9,7 & 414 & 16.0 & 0 & 0,0 \\
\hline saúde & 69 & 9,0 & 346 & 13,3 & 2 & 5,9 \\
\hline assistência social & 22 & 2,9 & 158 & 6,1 & 0 & 0,0 \\
\hline cultura, patrimônio histórico e artístico ${ }^{|3|}$ & 20 & 2,6 & 141 & 5,4 & 3 & 8,8 \\
\hline $\begin{array}{l}\text { formaçăo de mão-de-obra, geração de } \\
\text { emprego e renda }\end{array}$ & 37 & 4,8 & 106 & 4,1 & 0 & 0,0 \\
\hline participação popular & 25 & 3,3 & 81 & 3,1 & 0 & 0,0 \\
\hline $\begin{array}{l}\text { desenvolvimento regional e local em bases } \\
\text { sustentáveis }\end{array}$ & 24 & 3,1 & 73 & 2,8 & 6 & 17,6 \\
\hline limpeza pública & 12 & 1,6 & 67 & 2,6 & 2 & 5.9 \\
\hline idoso & 12 & 1,6 & 67 & 2,6 & 0 & 0,0 \\
\hline lazer e esportes ${ }^{|3|}$ & 3 & 0,4 & 63 & 2,4 & 0 & 0,0 \\
\hline deficiente & 44 & 5,8 & 43 & 1,7 & 0 & 0,0 \\
\hline saneamento & 26 & 3,4 & 37 & 1,4 & 0 & 0,0 \\
\hline segurança pública e policiamento & 54 & 7,1 & 4 & 0,2 & 0 & 0,0 \\
\hline demais áreas & 235 & 30,8 & 564 & 21,7 & 20 & 58,8 \\
\hline $\begin{array}{l}\text { Inclui iniciativas interestaduc } \\
\text { Inclui iniciativas intermunic }\end{array}$ & do Distrit & deral. & & & & \\
\hline $\begin{array}{l}\text { Em 1996, havia uma área, } \\
\text { dados dessa área foram incl }\end{array}$ & $\begin{array}{l}\text { ura, Lazer } \\
\text { s em Cult }\end{array}$ & $\begin{array}{l}\text { Esportes, } \\
\text { Patrimór }\end{array}$ & $\begin{array}{l}\text { foi desn } \\
\text { Histórico }\end{array}$ & $\begin{array}{l}\text { brada a } \\
\text { rtístico, }\end{array}$ & $\begin{array}{l}\text { de } 199 \\
\text { realizaçăc }\end{array}$ & $\begin{array}{l}\text { ortanto, os } \\
\text { tabela. }\end{array}$ \\
\hline
\end{tabular}

Analisando mais de perto os programas, projetos e atividades inscritos nos cinco anos de Programa GESTÃO PÚBLICA E CIDADANIA, é possivel identificar algumas tendências de mudança de atuação dos governos subnacionais na área social (Farah, 2000) ${ }^{\circ}$. Segundo Farah, estas tendências se articulam em torno de dois eixos principais: novas politicas sociais e novas formas de gestão.

Dentro do primeiro eixo, ou seja, novas políticas (conteúdo das políticas), as principais tendências são:

\footnotetext{
- Além desta análise realizada por Marta Ferreira Santos Farah, vice-diretora do Programa GESTÃO PÚBLICA E CIDADANIA, pesquisadores, professores e alunos de pós-graduação têm escrito artigos e trabalhos que são publicados pelo próprio Programa - ver Cadernos Gestão Pública e Cidadania, volumes 1 a 18 (Publicações, p. 205).
} 
- Mudanças na concepção sobre a natureza dos serviços prestados. Neste sentido podemos apontar as ações da área de saúde, que têm enfatizado a dimensão preventiva, substituindo o enfoque curativo que prevalecia no setor;

- Novas respostas a novos desafios. Por exemplo, na área da educação, onde, além da preocupação constante com a universalização do ensino, aparecem iniciativas cujos objetivos são combater a evasão escolar e a repetência e melhorar a qualidade de ensino;

- Ampliação do espaço da cidadania. Aqui está aparecendo uma tendência de governos locais em atender segmentos da população, antes esquecidos pelo poder público. É o caso dos programas voltados aos portadores de deficiência, às pessoas da terceira idade, à mulher, à comunidade negra e às crianças e adolescentes, que têm como referência o Estatuto da Criança e Adolescente - ECA - de 1990;

- Ampliação da esfera de ação do governo local. Trata-se, por exemplo, dos programas de geração de emprego e renda e de desenvolvimento local, os quais não faziam parte da preocupação dos governos subnacionais, sobretudo os municipais.

O outro eixo de mudança refere-se às novas formas de gestão, ou seja, à provisão e gestão de serviços públicos e aos processos de formulação e implementação das políticas. Dentre as tendências de inovação, podem ser citadas:

- Mudanças na relação entre Estado e Sociedade Civil. Desde os anos 80, há uma preocupação em incluir novos atores na formulação e implementação das políticas públicas. "Propostas de reforma de diversos setores da política social, como educação, saúde, habitação, assistência social, cultura, entre outras, incluiram, a partir de então, como um dos seus componentes centrais, a questão da participação popular, entendida como o exercício pleno de direitos de cidadania.";

- Articulação de diferentes niveis de governo, entre diversas administrações de mesmo nível e articulação intersetorial. Aqui destacam-se os inúmeros consórcios intermunicipais, as parcerias entre níveis de governos diferentes (governos municipais, estaduais e federais) e as ações integradas e intersetoriais;

- Gestão de programas e politicas estatais e da própria máquina pública. Esta dimensão gerencial aparece tanto em programas que abrangem toda a administração pública, como em programas setoriais (áreas de educação, saúde, saneamento etc.). "Os programas e projetos se voltam para a modernização da gestão, sendo possivel identificar, entre as iniciativas analisadas, os seguintes eixos de mudança: a) descentralização e democratização da gestão e do processo decisório, com ênfase à participação do servidor público nestes processos; b) descentralização das estruturas de decisão em direção à unidade prestadora do serviço, buscando uma maior proximidade em relação 
ao usuário e aos cidadãos; c) formação e qualificação de servidores; d) valorização do funcionalismo, através de melhoria de condições de trabalho e de melhoria salarial; e) reforço a sistemas de apoio a decisões - banco de dados, estatísticas gerenciais etc.; f) introdução de sistemas de avaliação de desempenho; g) adoção de programas de qualidade e produtividade; $h$ ) redução de custos."

As iniciativas inscritas nos cinco anos de Programa GESTÃo PÚBLICA E CIDADANIA confirmam a atuação dos governos subnacionais como parte de um processo amplo de Reforma do Estado, conseqüência da democratização do país e da crise fiscal que vêm ocorrendo deste a década de 80. "Seja como resultado de um esforço deliberado pela descentralização de competências e atribuições, seja pela descentralização de recursos a partir da Constituição de 1988, seja sob a influência da agenda descentralizadora das agências multilaterais, seja ainda como fruto da dinâmica democrática e da maior proximidade dos governos locais com relação às necessidades da população, 0 fato é que os governos estaduais e, sobretudo, os municipais estão promovendo um processo de reforma da ação do Estado na área social, rompendo com algumas das características da intervenção estatal no Brasil nesta área." (Farah, 2000).

Buscou-se com este texto apresentar alguns dados do quinto Ciclo de Premiação GESTÃo PÚBLICA E CIDADANIA e comparações com os quatro Ciclos anteriores. Esperamos trazer para o conhecimento do público informações gerais sobre os Ciclos de Premiação, estimulando, inclusive, pesquisas futuras mais aprofundadas.

\section{Bibliografia consultada}

BELTRÃO, Ricardo Ernesto Vasquez; FARAH, Marta Ferreira Santos \& LACZYNSKI, Patrícia. "Um primeiro olhar sobre o Ciclo de Premiação 1998". In: BELTRÃO, Ricardo Ernesto Vasquez; MAURO, Carlos Eduardo Evangelisti \& LACZYNSKI, Patrícia (org.). Cadernos Gestão Pública e Cidadania (A administração pública brasileira inovando a forma de governar: apresentação dos 631 programas inscritos no Ciclo de Premiação 1998). São Paulo: EAESP-FGV, v.12, 1998, p.7-19.

FARAH, Marta Ferreira Santos. Governo Local, Políticas Públicas e Novas Formas de Gestão Pública no Brasil . Organizações e Sociedade, Salvador: v.7, n. 17, jan./abr.2000, p.59-86.

FARAH, Marta Ferreira Santos \& BELTRÃO, Ricardo Ernesto Vasquez. "Perfil dos programas inscritos em 1997". In: BELTRÃO, Ricardo Ernesto Vasquez (org.). Cadernos Gestão Pública e Cidadania (A administração pública brasileira inovando a forma de governar: apresentação dos 297 programas inscritos no Ciclo de Premiação 1997). São Paulo: EAESP-FGV, v.8, 1996, p.7-14.

FARAH, Marta Ferreira Santos. "Que está mudando na gestão pública brasileira? Tendência sugeridas pelos programas inscritos". In: BELTRÃO, Ricardo Ernesto Vasquez (org.). Cadernos Gestão Pública $e$ 
Cidadania (A administração pública brasileira inovando a forma de governar - Ciclo de Premiação 1996). São Paulo: EAESP-FGV, v.1, 1996, p.7-16.

FUNDAC̣ÃO INSTITUTO BRASILEIRO DE GEOGRAFIA E ESTATÍSTICA - IBgE. Contagem Populacional 1996. Rio de Janeiro: IBGE, 1996.

LACZYNSKI, Patrícia, BELTRÃO, Ricardo Ernesto Vasquez \& FARAH, Marta Ferreira Santos. "Uma breve análise do Ciclo de Premiação 1999". In: LACZYNSKI, Patrícia., BARACCHINI, Sabrina Addison \& BELTRÃO, Ricardo Ernesto Vasquez (org.). Cadernos Gestão Pública e Cidadania (A administração pública brasileira inovando a forma de governar: apresentação dos 888 programas inscritos no Ciclo de Premiação 1999). São Paulo: EAESP-FGV, v.16, 1999, p.7-16.

SPINK, Peter. The Rights Approach to Local Public Management: experiences from Brazil. Revista de Administração de Empresas. São Paulo: v.40, n.3, jul./set.2000, p.45-65.

SPINK, Peter. Gestão Municipal faz escola: aprendendo com os governos locais. Cadernos Adenauer: os municípios e as eleições de 2000. São Paulo: n.4, jun.2000.

SPINK, Peter; CLEMENTE, Roberta \& KEPPKE, Rosane. Governo local: o mito e as novas práticas de governança. Revista de Administração. São Paulo: v.34, n.1, jan./mar.1999, p.61-9. 